\title{
ON THE LOCAL SEVERI PROBLEM
}

\section{ROBERT TREGER}

Introduction. We study plane curves with singularities. Let $\mathbf{P}^{N}$ be the projective space parametrizing plane curves of degree $n(N=n(n+3) / 2)$. Let $V(n, g) \subset \mathrm{P}^{N}$ be the locus of reduced irreducible plane curves of degree $n$ and (geometric) genus $g$, and $l \subset \mathbf{P}^{2}$ a fixed line. Following Zariski [7], we consider the subvariety $Z(n, g) \subset \overline{V(n, g)}$ of curves which contain $l$ as a component. The purpose of this note is to study $Z(n, g)$ and prove the following

THEOREM. Let $\mathscr{E}(n, g)$ be a branch of $\overline{V(n, g)}$ through a point of $Z(n, g)$ corresponding to a reduced curve. Then the general members of $\mathscr{E}(n, g) \cap$ $Z(n, g)$ have only nodes as singularities.

It is well known (cf. Severi $[\mathbf{5}, \S 11]$ ) that this Theorem implies the following fundamental result of Harris.

COROLLARY (HARRIS [3]). $V(n, g)$ is irreducible.

In the case when $L \in \mathscr{E}(n, g) \cap Z(n, g)$ is a union of $n$ distinct lines passing through a point, our theorem is a realization of Severi's attempt to prove that $L$ can be regenerated to a reducible nodal curve of $\mathscr{E}(n, g)[5, \S 11$, p. 344]. The idea of using decreasing induction on $g$ and equations of curves in the proof was suggested in Zariski [7]. On the other hand, Harris [3] and Ran [4] use the degeneration method in their treatment of plane curves.

Proof of Theorem. We set $d=(n-1)(n-2) / 2-g$ and $\nu(n, d)=$ $\operatorname{dim} V(n, g)=3 n+g-1([5, \S 11],[6])$. Let $\Sigma_{n, d} \subset \mathbf{P}^{N} \times \operatorname{Sym}^{d}\left(\mathbf{P}^{2}\right)$ be the closure of the locus of irreducible curves of degree $n$ with $d$ nodes and no other singularities, and $\pi_{N}$ the projection to $\mathbf{P}^{N}$. Given a pair consisting of a reduced curve $E \in \overline{V(n, g)}$ and a branch of $\overline{V(n, g)}$ through the curve, one can define, via $\pi_{N}$, an element of $\operatorname{Sym}^{d}\left(\mathbf{P}^{2}\right)$, called the cycle of assigned singularities of the pair. Our basic tool is the dimension-theoretic characterization of maximal families of nodal curves by Arbarello and Cornalba [1] and Zariski [6] and its generalization by Harris [3, Proposition 2.1].

Let $C$ be a general member of $\mathscr{E}(n, g) \cap Z(n, g)$. We will prove that $C$ is nodal and all its unassigned nodes lie on $l$ for every choice of a branch of $\mathscr{E}(n, g)$ through $C$.

LEMMA. For $d \leq 3, \Sigma_{n, d}$ is irreducible and unibranch.

ProOF OF THE LEMMA. Let $\Sigma^{\prime}, \Sigma^{\prime \prime} \subset \Sigma_{n, d}$ be components such that a general member of $\Sigma^{\prime}$ has $d$ nodes in general position. A dimension count

Received by the editors December 16, 1987.

1980 Mathematics Subject Classification (1985 Revision). Primary 14H10, 14 H45. 
shows that a general member of $\Sigma^{\prime} \cap \Sigma^{\prime \prime}$ is a reduced curve with $d$ assigned singular points. Therefore $\Sigma_{n, d}=\Sigma^{\prime}$. The second assertion follows from the unibranchness of $\operatorname{Sym}^{d}\left(\mathbf{P}^{2}\right)$.

Step 1 . Let $d \leq 3$. We regenerate $C$ to a nodal curve $F=l+F^{\prime}$ having the same number of irreducible components and the same genus as $C$. We take a branch of $\mathscr{E}(n, g)$ through $C$ and consider the corresponding cycle of assigned singularities $\sum d_{t} P_{t}$. For every $t$, we choose $d_{t}$ assigned nodes of $F$ in the vicinity of $P_{t}$. The curve $F$ with the $d=\sum d_{t}$ assigned nodes determines a branch of $\overline{V(n, g)}$ through $C$. By the lemma, it coincides with the branch of $\mathscr{E}(n, g)$, chosen above.

Step 2. We assume $d \geq 4$ and the theorem is true for smaller $d$ 's. If $C=l+C^{\prime}$ has no assigned singularities on $l$ (for a branch of $\mathscr{E}(n, g)$ ), then $C^{\prime}$ is moving in the family of dimension $\leq \nu(n-1, d)=\nu(n, d)-n-1$. Since $Z(n, g)$ is defined by $n+1$ equations in $\mathscr{E}(n, g)[7$, p. 470], the inequality is, in fact, equality and $C$ is nodal.

Step 3. We now assume that $C$ has assigned singularities on $l$. Let $f(X, Y, Z)=\Sigma a_{j k} X^{j} Y^{k} Z^{n-j-k}$ be an equation of a curve of $\mathscr{E}(n, g)$. We have chosen our coordinate system in $\mathbf{P}^{2}$ so that $l=\{X=0\}$ and all the singularities of the curves of $\mathscr{E}(n, g)$ lie in $\mathbf{P}^{2} \backslash\{Z=0\}$. Moreover, the following constructions take place in a neighborhood of a general member $D$ of $\mathscr{E}(n, g) \cap Z(n, g) \cap\left(a_{00}=a_{10}=a_{01}=0\right\}$. We assume $D$ is a specialization of $C$, and it has an assigned singularity at (0:0:1). By abuse of notation we denote by $\mathscr{E}(n, g)$ a fixed new branch of $\overline{V(n, g)}$ through $D$, contained in the original branch.

Let $\mathscr{A}_{g}^{0} \subset \mathscr{E}(n, g)$ be the subfamily of curves having a node at (0:0:1). It has codimension 2 in $\mathscr{E}(n, g)$ and its general members are irreducible nodal curves with $d$ nodes. For $i \geq 1$, the components of $\mathscr{A}_{g}^{i}=\mathscr{A}_{g}^{i-1} \cap\left\{a_{0 n-i+1}=\right.$ $0\}$ have codimension $\leq i+2$ in $\mathscr{E}(n, g)$ and consist of curves having intersection multiplicity at least $i$ with $l$ at $(0: 1: 0)$. If a general member of $\mathscr{A}_{g}^{i}$ does not contain $l$ as a component, then a dimension count shows that it has intersection multiplicity $i$ with $l$ at (0:1:0) and only $d$ singular points which are nodes: we blow up (0:0:1) and $i$ times $(0: 1: 0)$ in the direction of $l$, and apply [3, Proposition 2.1].

Step 4. Let $E$ be a general member of $\mathscr{E}(n, g)$ and $Q \in E$ a node distinct from (0:0:1). Moreover, if $D$ has an assigned (with respect to $\mathscr{E}(n, g)$ ) singular point outside $l$, then we assume $Q$ tends to this point; the second choice is a node $Q \in E$ which does not tend to $(0: 0: 1)$. Let $\mathscr{E}(n, g+1) \subset \overline{V(n, g+1)}$ $(\mathscr{E}(n, g) \subset \mathscr{E}(n, g+1))$ be the branch through $D$ obtained by considering $Q$ as virtually nonexistent. We can define, as above, the subfamilies $\mathscr{A}_{g+1}^{i}$ of $\mathscr{E}(n, g+1)$. Let $m+1$ be the first integer such that a general member of $\mathscr{A}_{g}{ }^{m+1}$ contains $l$ as a component.

Case 1. The general members of $\mathscr{A}_{g+1}^{m+1}$ do not contain $l$ as a component. We get $\operatorname{dim} \mathscr{A}_{g+1}^{m+2}=\operatorname{dim} \mathscr{A}_{g}^{m+1}$. By the induction hypothesis we get that $D$ is nodal.

Case 2. A general member $F$ of $\mathscr{A}_{g+1}^{m+1}$ contains $l$ as a component. Then $F=l+F^{\prime}$ is nodal. A dimension count shows that $D=l+D^{\prime}$ can have at 
most one non-nodal singularity. Moreover, if $g\left(F^{\prime}\right) \neq g\left(D^{\prime}\right)$ then $D$ is nodal. If $D$ is not nodal and $g\left(F^{\prime}\right)=g\left(D^{\prime}\right)$, then the singular points of $F$ and $D$ are on $l$. Hence $F^{\prime}$ is smooth and $D$ has one tacnode. We can assume the node $Q$ tends to a node $Q^{*} \in D$. Let $\mathscr{H} \subset \Sigma_{n, 1}$ be the branch through $\left(D, Q^{*}\right)$. By [2, Exp. XIII, §2],

$$
\mathscr{B}=\mathscr{E}(n, g+1) \cap \pi_{N}(\mathscr{H})
$$

is 1-connected. If $\mathscr{B}=\mathscr{E}(n, g)$, we are done. If $\mathscr{B} \neq \mathscr{E}(n, g)$, we choose a component $\mathscr{C}$ of $\mathscr{B}$ such that a component $\mathscr{U}$ of $\mathscr{C} \cap \mathscr{E}(n, g)$ has dimension $\nu(n, d+1)$. Let $G$ be a general member of $\mathscr{U}$. By the deformation theory, we get $g(G) \leq g-1$. Therefore $G$ is nodal with $d+1$ nodes, two of which tend to the tacnode. By intersecting $\mathscr{U}$ with the branches of $\pi_{N}\left(\Sigma_{n, 1}\right)$ corresponding to the unassigned nodes of $D$, we derive that $D$ is not a general curve.

REMARKS. A dimension count shows that the number of unassigned singularities of $C$ is equal to $m$.

As in the lemma, for $d \leq n(n+3) / 6$ and $(n, d) \neq(6,9)$, the unibranchness of $\Sigma_{n, d}$ follows from the irreduciblity. One can give another proof that $\Sigma_{n, d}$ is irreducible in that range using the following general result (see a conjecture in [7, p. 479]): Let $E$ be a general member of an irreducible subfamily $S$ of $\overline{V(n, g)}(0 \leq g \leq(n-1)(n-2) / 2)$. If $\operatorname{dim} S \geq \nu(n, d)-3$, then $E$ is reduced. If $S$ consists of nonreduced curves and has the maximal dimension, then a general member of $S$ is a union of an irreducible nodal curve and a general double line.

\section{REFERENCES}

1. E. Arbarello and M. Cornalba, Su una proprietà notevole dei morfismi di una curva a moduli generali in una spazio proiettivo, Rend. Sem. Mat. Univ. Polytecn. Torino 38 (1980), 87-99.

2. A. Grothendieck et al., Cohomologie locale des faisceaux cohérents et Théorèmes de Lefschetz locaux et globaux, North-Holland, Amsterdam, 1968.

3. J. Harris, On the Severi problem, Invent. Math. 84 (1986), 445-461.

4. Z. Ran, On nodal plane curves, Invent. Math. 86 (1986), 529-534.

5. F. Severi, Vorlesungen über algebraische Geometrie, Anhang F, Teubner, Leipzig, 1921.

6. O. Zariski, Algebraic systems of plane curves, Amer. J. Math. 104 (1982), 209-226.

7. with nodes, Arithmetic and Geometry, Vol. II, Papers dedicated to I. R. Shafarevich (M. Artin and J. Tate, eds.), Birkhäuser, Boston, 1983, pp. 465-481.

Department of Mathematics, QUeen's University, Kingston, ONtario, CANADA K7L 3N6 
